\title{
The Landscape of Emerging Randomized Clinical Trial Evidence for COVID-19 Disease Stages: A Systematic Review of Global Trial Registries
}

This article was published in the following Dove Press journal: Infection and Drug Resistance

\author{
Alison Dillman' \\ Michael J Zoratti ${ }^{2}$ \\ Jay JH Park ${ }^{3}$ \\ Grace Hsu $\mathbb{D}^{2}$ \\ Louis Dron ${ }^{2}$ \\ Gerald Smith ${ }^{2}$ \\ Ofir Harari $\mathbb{D}^{2}$ \\ Craig R Rayner ${ }^{4}$ \\ Noor-E Zannat ${ }^{2}$ \\ Alind Gupta ${ }^{2}$ \\ Eric Mackay ${ }^{5}$ \\ Paul Arora ${ }^{6}$ \\ Zelyn Lee ${ }^{7}{ }^{7}$ \\ Edward J Mills ${ }^{2}$ \\ 'School of Public Health, Faculty of \\ Medicine, Imperial College London, \\ London, England; ${ }^{2}$ Department of Health \\ Research Methods, Evidence, and Impact, \\ McMaster University, Hamilton, Canada; \\ ${ }^{3}$ Department of Experimental Medicine, \\ University of British Columbia, \\ Vancouver, British Columbia, Canada; \\ ${ }^{4}$ Monash Institute of Pharmaceutical \\ Sciences, Monash University, Melbourne, \\ Australia; ${ }^{5}$ Department of Statistical \\ Sciences, University of Toronto, Toronto, \\ Canada; ${ }^{6}$ Dalla Lana School of Public \\ Health, University of Toronto, Toronto, \\ Canada; ${ }^{7}$ Department of Physiology \& \\ Department of Neuroscience, University \\ of Toronto, Toronto, Canada
}

Correspondence: Jay JH Park $\mathrm{Tel}+$ I 604 294-3823

Email jayhparkI@alumni.ubc.ca
Purpose: A multitude of randomized controlled trials (RCTs) have emerged in response to the novel coronavirus disease (COVID-19) pandemic. Understanding the distribution of trials among various settings is important to guide future research priorities and efforts. The purpose of this review was to describe the emerging evidence base of COVID-19 RCTs by stages of disease progression, from pre-exposure to hospitalization.

Methods: We collated trial data across international registries: ClinicalTrials.gov; International Standard Randomised Controlled Trial Number Registry; Chinese Clinical Trial Registry; Clinical Research Information Service; EU Clinical Trials Register; Iranian Registry of Clinical Trials; Japan Primary Registries Network; German Clinical Trials Register (up to 7 October 2020). Active COVID-19 RCTs in international registries were eligible for inclusion. We extracted trial status, intervention(s), control, sample size, and clinical context to generate descriptive frequencies, network diagram illustrations, and statistical analyses including odds ratios and the Mann-Whitney $U$-test.

Results: Our search identified 11503 clinical trials registered for COVID-19 and identified 2388 RCTs. After excluding 45 suspended RCTs and 480 trials with unclear or unreported disease stages, 1863 active RCTs were included and categorized into four broad disease stages: pre-exposure $(n=107)$; post-exposure $(n=208)$; outpatient treatment $(n=266)$; hospitalization, including the intensive care unit $(\mathrm{n}=1376)$. Across all disease stages, most trials had two arms ( $\mathrm{n}=1500 / 1863,80.52 \%)$, most often included (hydroxy)chloroquine ( $\mathrm{n}=271 /$ $1863,14.55 \%)$ and were US-based ( $n=408 / 1863,21.90 \%)$. US-based trials had lower odds of including (hydroxy)chloroquine than trials in other countries (OR: $0.63,95 \%$ CI: $0.45-0.90$ ) and similar odds of having two arms compared to other geographic regions (OR: $1.05,95 \%$ CI: $0.80-1.38$ ).

Conclusion: There is a marked difference in the number of trials across settings, with limited studies on non-hospitalized persons. Focus on pre- and post-exposure, and outpatients, is worthwhile as a means of reducing infections and lessening the health, social, and economic burden of COVID-19.

Keywords: COVID-19, novel coronavirus 2019, systematic review, randomized controlled trials

\section{Introduction}

The COVID-19 pandemic, caused by the novel severe acute respiratory syndromecoronavirus 2 (SARS-CoV-2), has mobilized the global health research community in an effort to rapidly identify preventative strategies and effective therapies. ${ }^{1}$ Indeed, we are witnessing an international research community aligned with 
a common goal. ${ }^{2,3}$ The substantial investments into treatment and prevention efforts have resulted in clinical trials emerging at an unprecedented rate. ${ }^{4,5}$ Yet, despite having this common goal, critics have highlighted limited collaboration and coordination among the research community. ${ }^{6-8}$ However, the scope of benefit to be realized from international coordination is substantial and proven in other wide-scale research projects, as evident in the rapid advancements in genomic medicine arising from the human genome project. ${ }^{9-11}$

The wealth of publicly available data on registered COVID-19 clinical trials provides an opportunity to describe and evaluate this emerging landscape. Public health professionals are interested in identifying differences in research focuses across the spectrum of COVID-19, from prophylaxis and early disease to hospitalization. ${ }^{12,13}$ Identification of potential gaps in research efforts may help guide researchers to areas requiring more focus, thereby encouraging new activities to better our understanding of COVID-19. To provide such an overview, we conducted a systematic review of registered randomized controlled trials (RCTs) to summarize and characterize research efforts with respect to the different stages of disease progression.

\section{Methods}

\section{Data Sources and Searches}

We generated a global database of COVID-19 research activities by identifying trials listed in international registries. $^{4}$ Specifically, we reviewed entries in "ClinicalTrials.gov", "International Standard Randomised Controlled Trial Number (ISRCTN) Registry", "Chinese Clinical Trial Registry", "Clinical Research Information Service - Republic of Korea", "European Union (EU) Clinical Trials Register", "Iranian Registry of Clinical Trials", “Japan Primary Registries Network”, and "German Clinical Trials Register". Searches were conducted using the search terms "novel coronavirus 2019 OR COVID-19 OR SARS-CoV-2" or database-specific tools to list COVID-19 registered trials, where available, in all clinical trial registries up to 7 October 2020 (Supplementary Table 1).

\section{Trial Selection}

In this review, we describe randomized trials of any active interventions for COVID-19. Randomization status was determined through self-reported methods and, where possible, the randomization masking procedure. Randomized diagnostic studies without active intervention components and trials with unclear or unreported disease stages were excluded. Suspended trials and studies with non-randomized designs were also excluded. Only trials registered in English were eligible for inclusion. A paired group of six reviewers screened for eligibility (Supplementary Table 2). Discrepancies in trial selection were resolved by a third investigator.

\section{Data Extraction}

Study details, including the trial identifier, recruitment status, interventions, study and site locations, number of study arms, blinding, planned sample size, eligibility criteria, outcomes, and disease stages represented were extracted from each registry entry. Disease stage was identified through study inclusion and exclusion criteria at enrolment. In this definition, outpatient studies enrolled patients who tested positive for COVID-19 but could not be hospitalized at baseline, and were identified as preexposure or post-exposure based upon exposure status at baseline. Studies with patients admitted to the hospital or intensive care unit at baseline were identified as such. Similarly, preventive trials required participants to test negative at baseline. Studies that recruited patients across multiple clinical settings (eg outpatients and prophylaxis in separate treatment arms) were counted across multiple categories.

\section{Data Synthesis and Analysis}

We describe the landscape of COVID-19 RCTs with respect to their clinical context, referring to whether participants were enrolled pre-exposure (PrEP), post-exposure (PEP), as outpatients, or when hospitalized or in the intensive care unit (ICU) as a result of COVID-19. Trial characteristics across the body of emerging evidence are summarized and the anticipated networks of evidence arranged by these clinical contexts are illustrated. We used the statistical software $\mathrm{R}$ version 4.0.2 to generate descriptive frequencies, network diagram illustrations, and statistical analyses. ${ }^{14}$ Network diagrams present interventions as nodes with lines representing a trial between the interventions (ie a direct comparison). ${ }^{15}$ We used odds ratios (ORs) for categorical outcomes and the MannWhitney $U$-test for continuous outcomes with two levels. Estimates of effect sizes are presented as ORs with associated 95\% confidence intervals (CIs) for categorical outcomes, and as mean ranks for continuous outcomes. 
The extracted data on active treatments were coded into treatment categories and the statistical software $\mathrm{R}$ version 4.0.2 was used to generate treatment network diagrams for each of the disease stages. Interventions for each included clinical trial were coded into broader treatment categories (eg umifenovir and favipiravir were categorized as antivirals). For illustrative purposes, select interventions that have received specific attention by the broader research community (eg (hydroxy)chloroquine, lopinavir/ritonavir, and remdesivir) were not collapsed into categories. Treatments that appeared in fewer than five unique trials were not included in the network diagrams.

\section{Results}

\section{Trial Registry Search and Trial Identification}

After reviewing a total of 11503 records across major international registries, we identified 2388 RCTs for COVID-19. We excluded 480 trials with unclear or unreported patient settings and 45 suspended RCTs to include 1863 RCTs in our review (Figure 1). Across these studies, 107 (5.74\%) and 208 (11.16\%) enrolled participants in the PrEP and PEP settings, respectively, with a further 266 $(14.28 \%)$ trials in the outpatient setting and 1376 (73.86\%) in patients hospitalized or in the ICU (Figure 2).

\section{Clinical Trial Characteristics}

Approximately half $(\mathrm{n}=980 / 1863,52.60 \%)$ of the included trials were listed as currently recruiting, 635 $(34.08 \%)$ were not currently recruiting, and 248 (13.31\%) had completed. The United States had the largest number of registered trials $(\mathrm{n}=408 / 1863,21.90 \%)$, followed by Iran $(n=243 / 1863,13.04 \%)$ and China $(n=223$ / $1863,11.97 \%)$. The median planned sample size across all trials was 100 participants (interquartile range [IQR]: 50, 308 ) and the median number of arms was two (IQR: 2, 2). The most frequently included intervention was (hydroxy) chloroquine ( $\mathrm{n}=271 / 1863,14.55 \%)$. A Mann-Whitney $U$-test showed that the trials conducted in the United States did not have important differences in sample size (median: 120, IQR: 50, 376, mean rank: 910.10) compared to trials conducted in other geographic regions (median: 100, IQR: 50, 300, mean rank: 855.73, $\mathrm{p}=0.095)$. United States-based clinical trials had lower odds of including (hydroxy)chloroquine for their clinical investigation than trials in other countries (OR: $0.63,95 \%$ CI: 0.45 to 0.90 ).
When we dichotomized the number of interventions as "two-arm trials" and "multi-arm trials" defined by trials having three or more arms, we found that United Statesbased trials had similar odds of having two arms compared to trials in other geographic regions (OR: $1.05,95 \% \mathrm{CI}$ : 0.80 to 1.38 ).

The distributions of the number of arms for trials of any interventions and for (hydroxy)chloroquine only, arranged by disease stage, are illustrated in Figure 3A and $\mathrm{B}$, respectively. The median number of intervention arms across all RCTs and RCTs treating with (hydroxy) chloroquine was two (IQR: 2,2) where one experimental intervention arm was compared to one other control arm. The proportion of two-arm studies across all trials was 80.52\% ( $\mathrm{n}=1500 / 1863)$. Between PEP $(\mathrm{n}=176 / 208$, $84.62 \%)$, outpatient $(\mathrm{n}=194 / 266,72.93 \%)$; and hospital or ICU settings $(n=1133 / 1376,82.34 \%)$, the proportion of two-arm trials were comparable. The PrEP disease stage had the lowest proportion of two-arm trials $(\mathrm{n}=62 / 107$, $57.94 \%$ ) compared to other stages; however, this is likely due to the lower number of trials being conducted for this disease stage. Among RCTs investigating (hydroxy)chloroquine, the proportion of two-arm studies was highest in the PrEP setting ( $\mathrm{n}=11 / 13,84.62 \%)$, followed by PEP ( $\mathrm{n}$ $=49 / 63,77.78 \%)$, the hospital or ICU $(\mathrm{n}=96 / 162$, $59.26 \%)$, and the outpatient setting $(\mathrm{n}=22 / 47,46.81 \%)$.

\section{Breakdown of Key Therapeutic Interventions}

The distribution of COVID RCTs and the enrolment target for the key therapeutic interventions for PEP, outpatients, and hospitalized settings are presented in Figure 4. In addition to (hydroxy)chloroquine, plasma-based therapy ( $\mathrm{n}=$ 151), lopinavir/ritonavir $(\mathrm{n}=90)$, azithromycin $(\mathrm{n}=79)$, tocilizumab $(\mathrm{n}=59)$, remdesivir $(\mathrm{n}=25)$, colchicine $(\mathrm{n}=$ 16), and dexamethasone $(\mathrm{n}=9)$ were the next top seven most frequently investigated interventions. (Hydroxy)chloroquine was included in $12.15 \%(n=13 / 107)$ of PrEP trials, $30.29 \%(n=63 / 208)$ of PEP trials, $17.67 \%(n=47 / 266)$ of trials in the outpatient setting, and $11.77 \%(n=162 / 1376)$ of trials in the hospital or ICU settings.

\section{Clinical Trials for Pre-Exposure Prophylaxis (PrEP)}

The treatment network for the PrEP setting consisted of 107 RCTs arranged into 12 treatment categories (Figure $5 \mathrm{~A})$. Ten studies $(9.35 \%)$ randomized patients to 

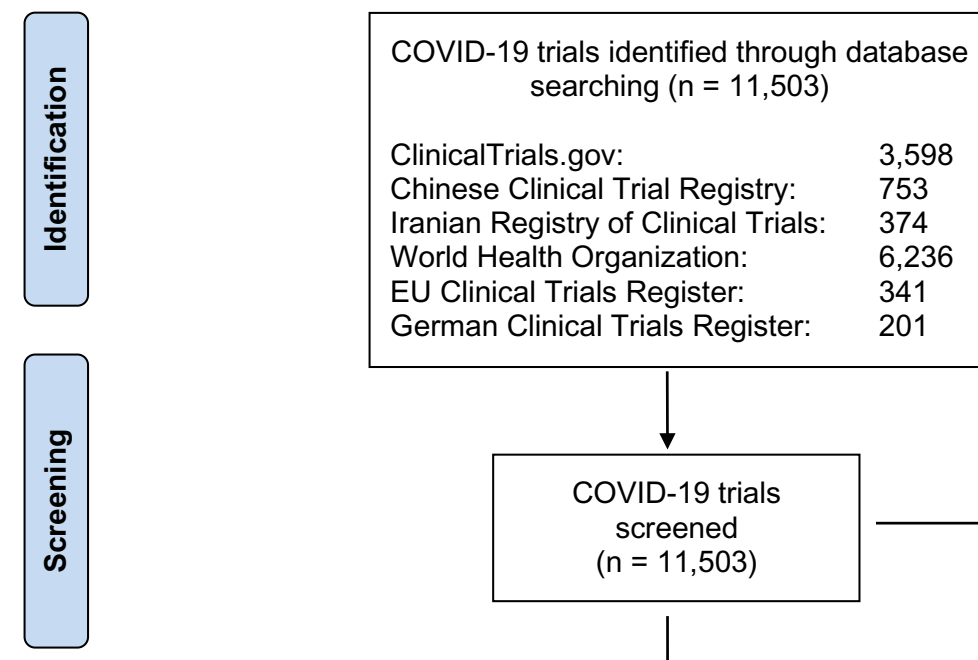

German Clinical Trials Register: 201
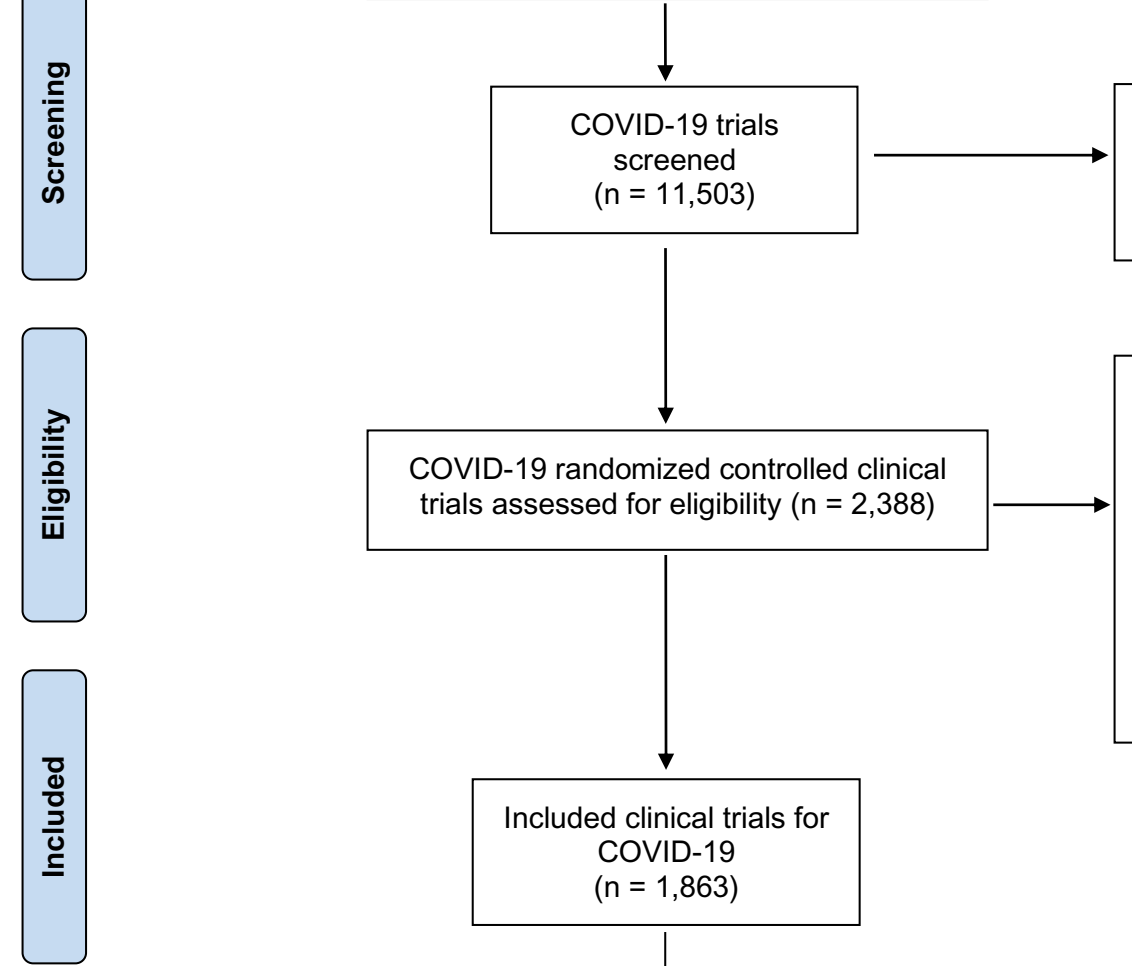

COVID-19 nonrandomized controlled trials excluded $(n=9,115)$

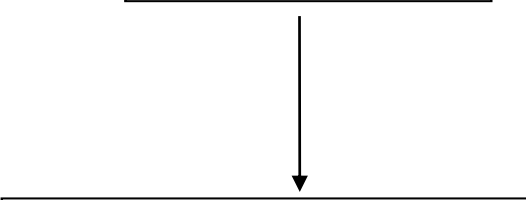

COVID-19 randomized controlled clinical trials assessed for eligibility $(n=2,388)$

Pre-exposure $(n=107)$
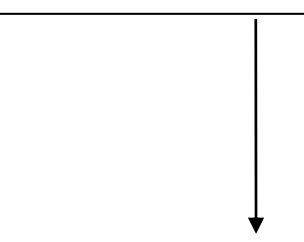

Population: 0 Interventions: 480 Comparators: 0 Outcomes: 0 Study design: 0 Trial status: $\quad 45$

Registered clinical trials excluded, with reasons $(n=525)$

Included clinical trials for COVID-19

$(n=1,863)$

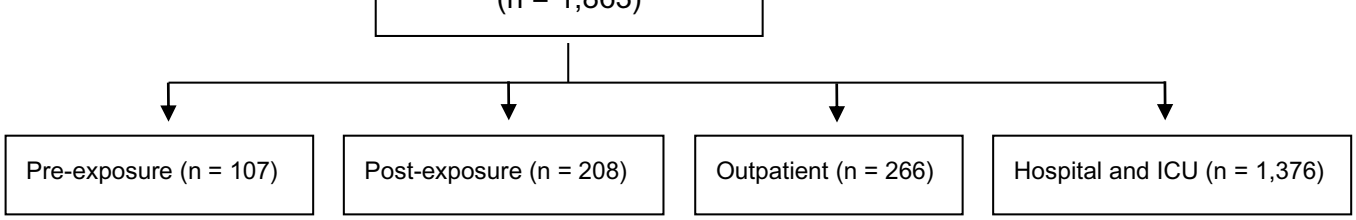

Figure I Study flowchart.

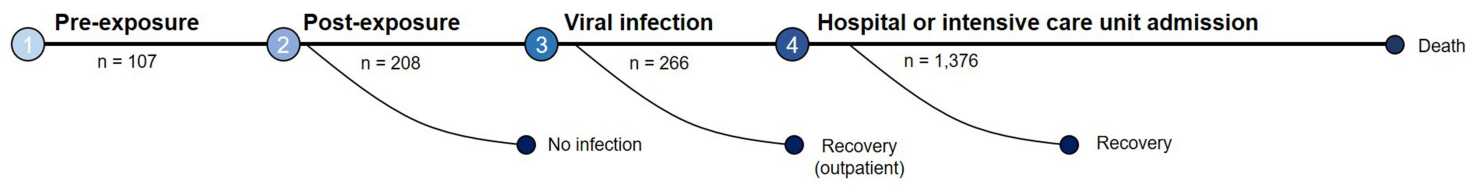

Figure 2 Randomised clinical trials at each stage of disease progression for COVID-19.

Notes: This figure illustrates broad disease stages of COVID-19 disease starting from pre-exposure to SARS-CoV-2, post-exposure, early infection in the outpatient setting, hospitalization and to advanced disease manifestation that requires intensive care.

(hydroxy)chloroquine monotherapy, the most common intervention category across trials in this setting (Figure 4). Three additional studies (2.80\%) listed a (hydroxy)chloroquine combination therapy with radiation therapy or zinc. The local standard of care was the most common control group ( $\mathrm{n}=66 / 107,61.68 \%$ ), representing a largely heterogeneous category of treatments and non-treatments (ie placebo) which varied across studies. The median number of arms for all trials and (hydroxy)chloroquine trials was two (IQR: 2, 3) (Figure 3).

Outcomes varied across PrEP trials, with nearly half reporting adverse events $(n=47 / 107,43.93 \%)$, followed by mortality ( $\mathrm{n}=11 / 107,10.28 \%$ ), and viral load or 
A

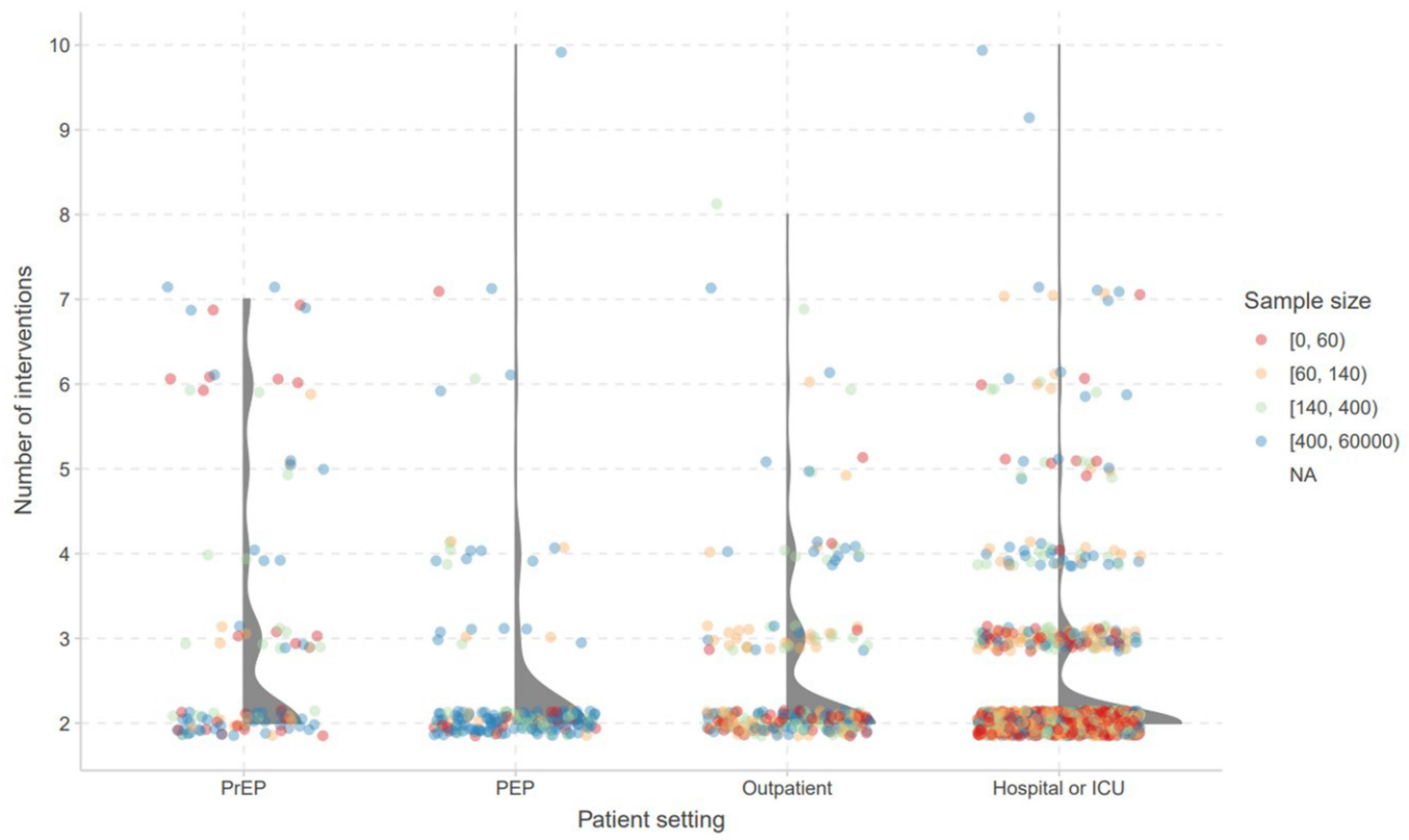

B

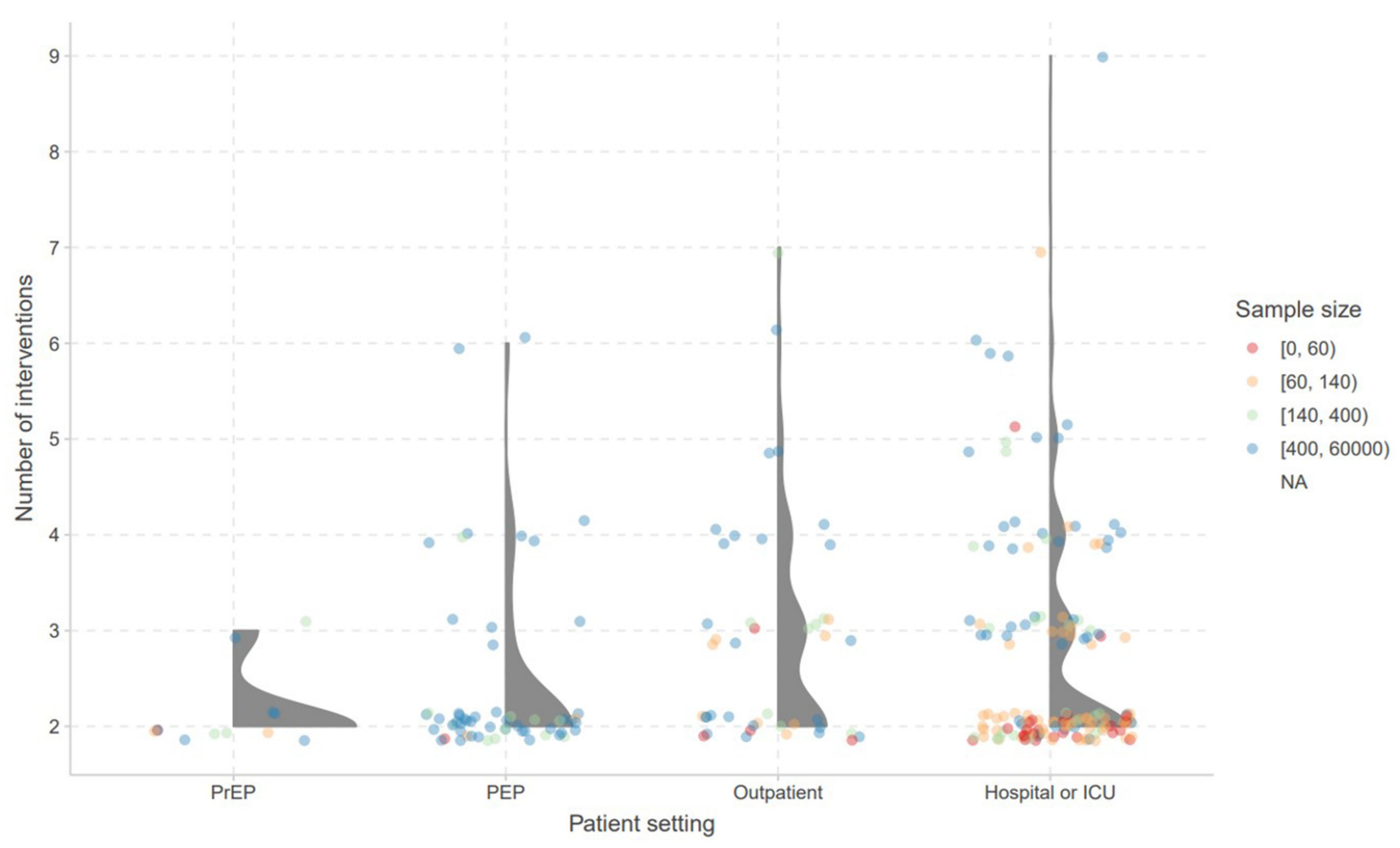

Figure 3 Distribution of number of arms for $(\mathbf{A})$ all and $(\mathbf{B})$ (hydroxy)chloroquine randomized clinical trials for COVID-19, arranged by clinical setting.

Notes: Each circle represents an individual trial. The colour of each circle represents the sample size: red - 0, 60; orange - 60, 140; green - I40, 400; blue - 400, 60,000 participants.

Abbreviations: PrEP, pre-exposure prophylaxis; PEP, post-exposure prophylaxis; ICU, intensive care unit.

clearance $(n=9 / 107,8.41 \%)$. Five $(4.67 \%)$ of the trials reported the incidence of hospitalization and eight (7.48\%) reported intensive care unit admission. Eighteen (16.82\%) trials were based in China, with 10 (9.35\%) in the United
States and $8(\mathrm{n}=7.48 \%)$ in the United Kingdom. Planned sample sizes in PrEP trials were higher than the overall average, with a median of 240 participants (IQR: 75, 1200). 


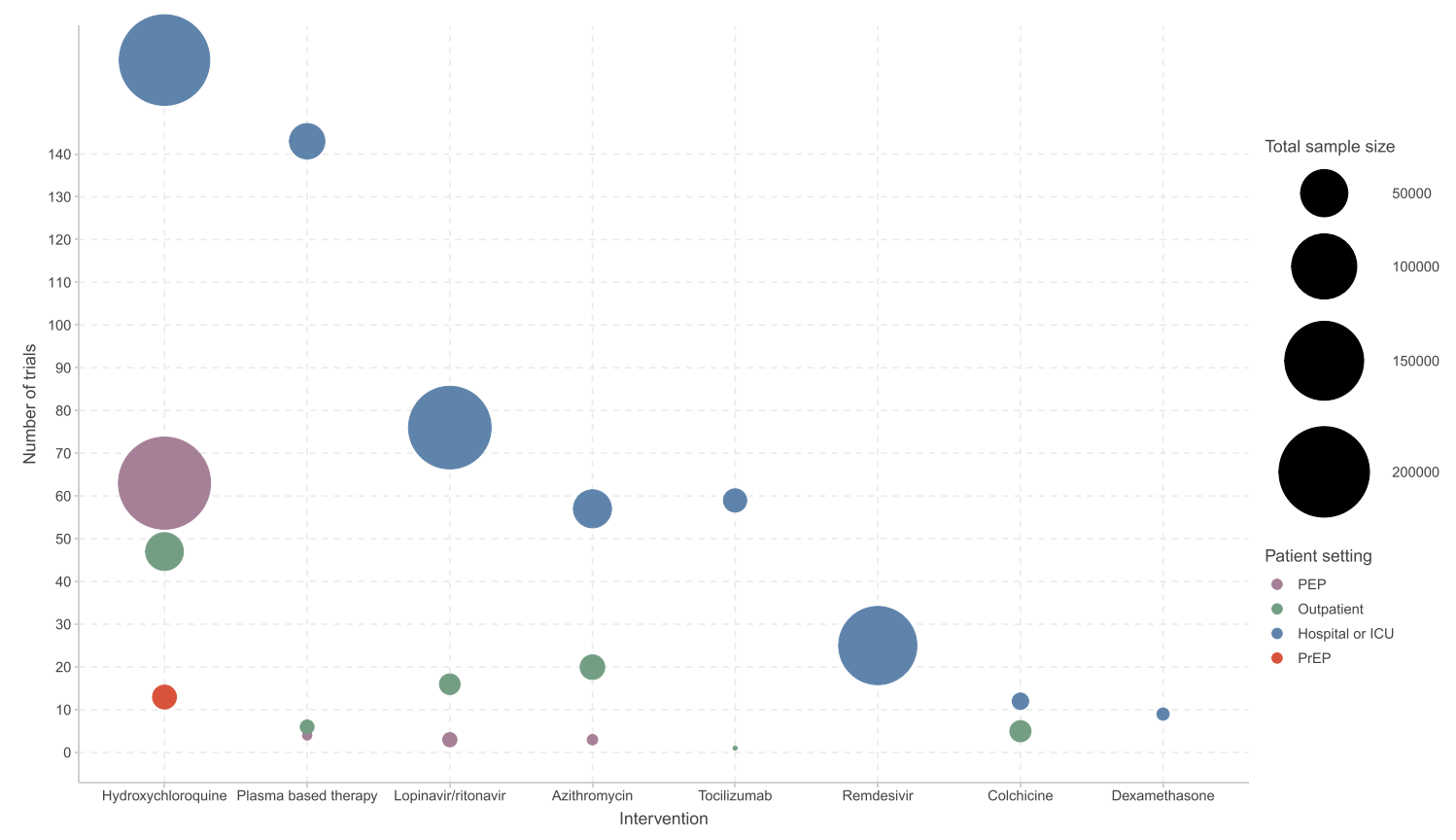

Figure 4 Distribution of COVID-19 randomized clinical trials and enrollment target for key drugs, arranged by clinical setting.

Notes: The size of each circle represents the total sample size (recruitment) of trials. Each colour represents the patient setting: purple - PEP; red - PrEP; green outpatient; blue - hospital or ICU.

Abbreviations: PEP, post-exposure prophylaxis; ICU, intensive care unit.

\section{Clinical Trials for Post-Exposure Prophylaxis (PEP)}

The treatment network for trials in the PEP setting consists of 19 treatment categories across the 208 RCTs (Figure 5B). Approximately a third $(\mathrm{n}=60 / 208,28.85 \%)$ of trials include (hydroxy)chloroquine monotherapy as an intervention arm (Figure 4). Three additional trials $(n=3 / 208$, $1.44 \%$ ) include (hydroxy)chloroquine combination therapy with azithromycin, zinc and nucleoside/nucleotide reverse transcriptase inhibitors. The majority of trials $(n=156 /$ $208,75.0 \%$ ) used local standard of care as the control group. The median number of arms across all trials and (hydroxy)chloroquine trials for PEP was two (IQR: 2, 2) (Figure 3).

Viral load or clearance was an outcome in approximately a third $(\mathrm{n}=60 / 208,28.85 \%)$ of trials, with 47 (22.60\%), 41 (19.71\%), 40 (19.23\%), and $30(14.42 \%)$ listing adverse events, hospitalization, mortality, and ICU admission as an outcome, respectively. India and the United States were the most represented countries, with $49(23.56 \%)$ and $46(22.12 \%)$ trials, respectively. Eleven trials $(5.29 \%)$ were reported in China and nine $(4.33 \%)$ in Iran. Planned sample sizes varied, with a median enrolment of 500 participants (IQR: 200, 1500).

\section{Clinical Trials for the Outpatient Setting} Twenty-five treatment categories were identified from 266 trials in the outpatient setting (Figure 6A). (Hydroxy)chloroquine monotherapy was the most common treatment $(\mathrm{n}=32 / 266,12.03 \%)$, followed by azithromycin $(\mathrm{n}=20 / 266,7.52 \%)$, lopinavir/ritonavir $(\mathrm{n}=$ $16 / 266,6.02 \%$ ) and (hydroxy)chloroquine combination therapy ( $n=15 / 266,5.64 \%$ ) (Figure 4$)$. The local standard of care remained the most common comparator group and was included in 206 (77.44\%) trials. Across all trials and (hydroxy)chloroquine trials in the outpatient setting, the median number of arms was two (IQR: 2, 3) (Figure 3).

Across the 266 RCTs, the need for hospitalization ( $\mathrm{n}=$ $90 / 266,33.83 \%)$, mortality $(\mathrm{n}=90 / 266,33.83 \%)$, and viral load or clearance $(n=89 / 266,33.46 \%)$ were the most commonly listed outcomes, followed by adverse events in $84(31.58 \%)$ trials, need for invasive mechanical ventilation $(\mathrm{n}=52 / 266,19.55 \%)$, and need for admission to the ICU ( $n=47 / 266,17.67 \%)$. Seventy $(26.32 \%)$ trials were conducted in the United States and 48 (18.05\%) trials were conducted in China. Average planned sample sizes were smaller than either the PrEP or PEP setting, with a median sample size of 156 participants (IQR: 80, 400). 
A

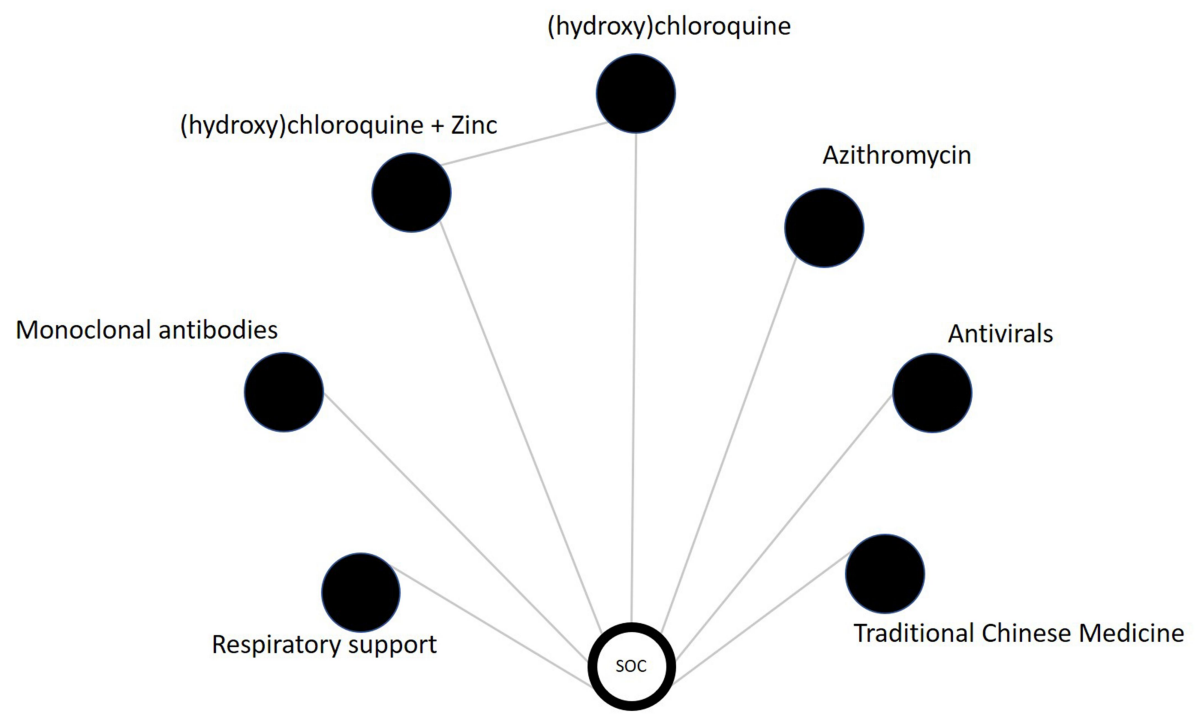

B

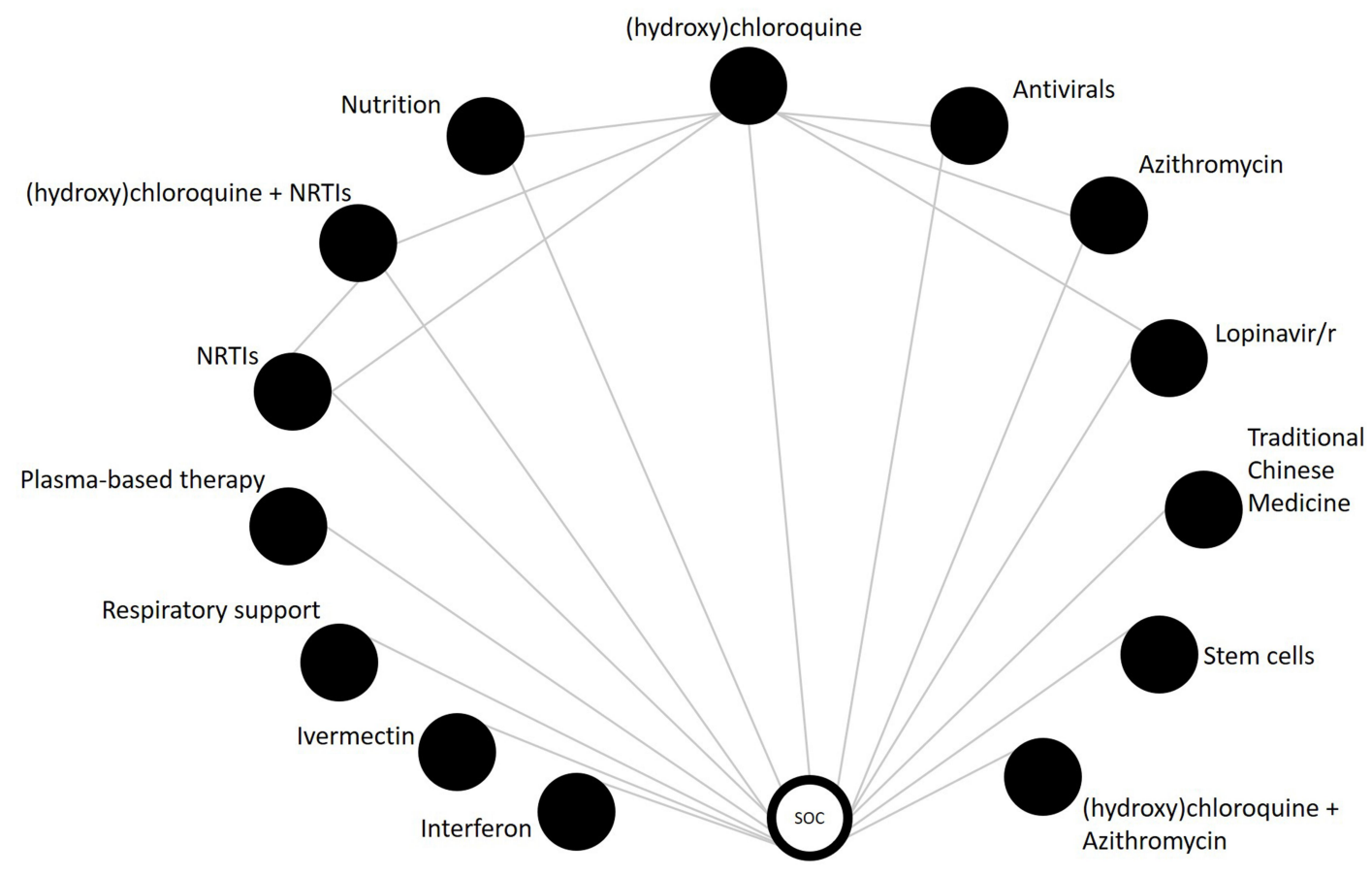

Figure 5 Treatment network for (A) pre-exposure and (B) post-exposure randomized clinical trials for COVID-19.

Notes: Each node (circle) represents an intervention and each line represents a direct comparison between different interventions.

Abbreviations: NRTIs, nucleoside reverse transcriptase inhibitors; Lopinavir/r, lopinavir/ritonavir.

\section{Clinical Trials for the Hospitalized and Intensive Care Unit Settings}

Forty treatment categories were identified across the 1376 RCTs in the hospitalized or ICU setting (Figure 6B). (Hydroxy)chloroquine was the most commonly investigated intervention $(\mathrm{n}=162 / 1376,8.70 \%)$, appearing in
$46(2.47 \%)$ trials as monotherapy and in $116(6.23 \%)$ trials in combination with other interventions, including lopinavir/ritonavir $(\mathrm{n}=76)$, azithromycin $(\mathrm{n}=57)$, and antivirals $(n=28)$ (Figure 4). Plasma-based and stem cell therapies were studied in this patient population and were described in $143(7.68 \%)$ and $67(3.60 \%)$ trials, respectively. The 
A

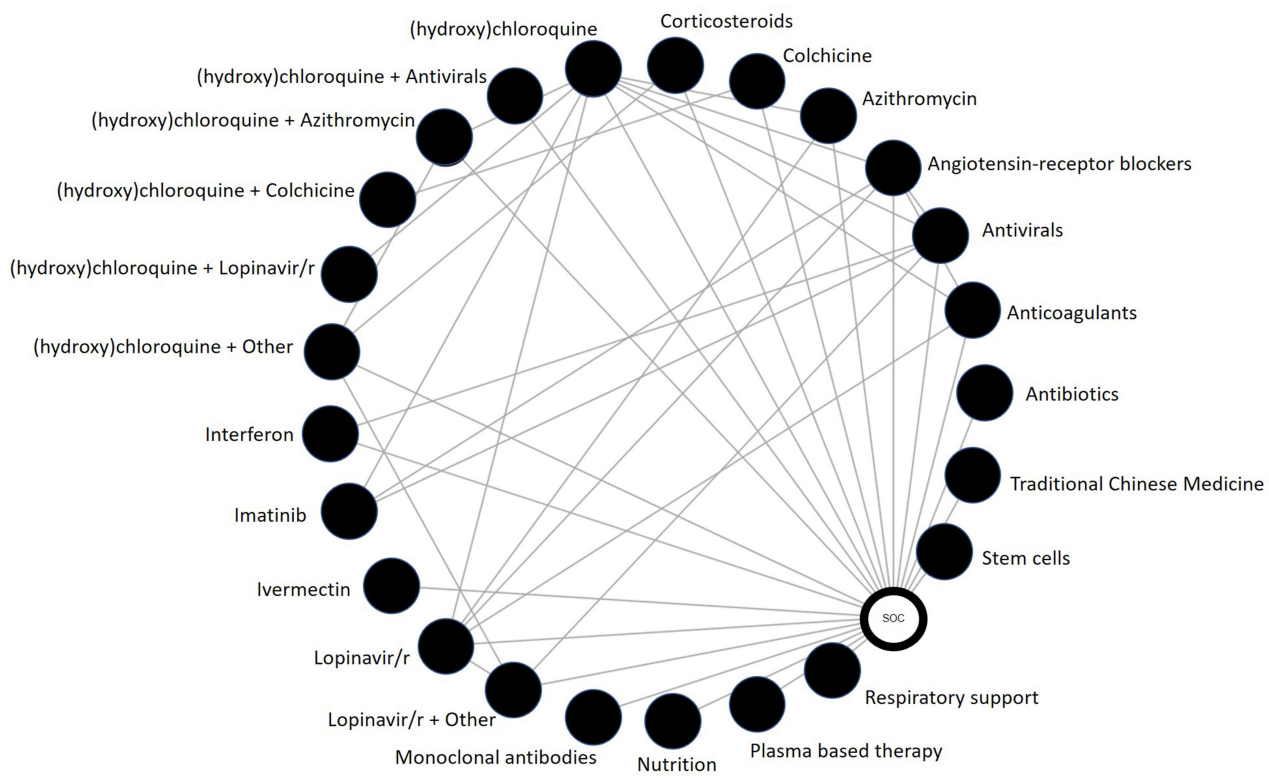

B

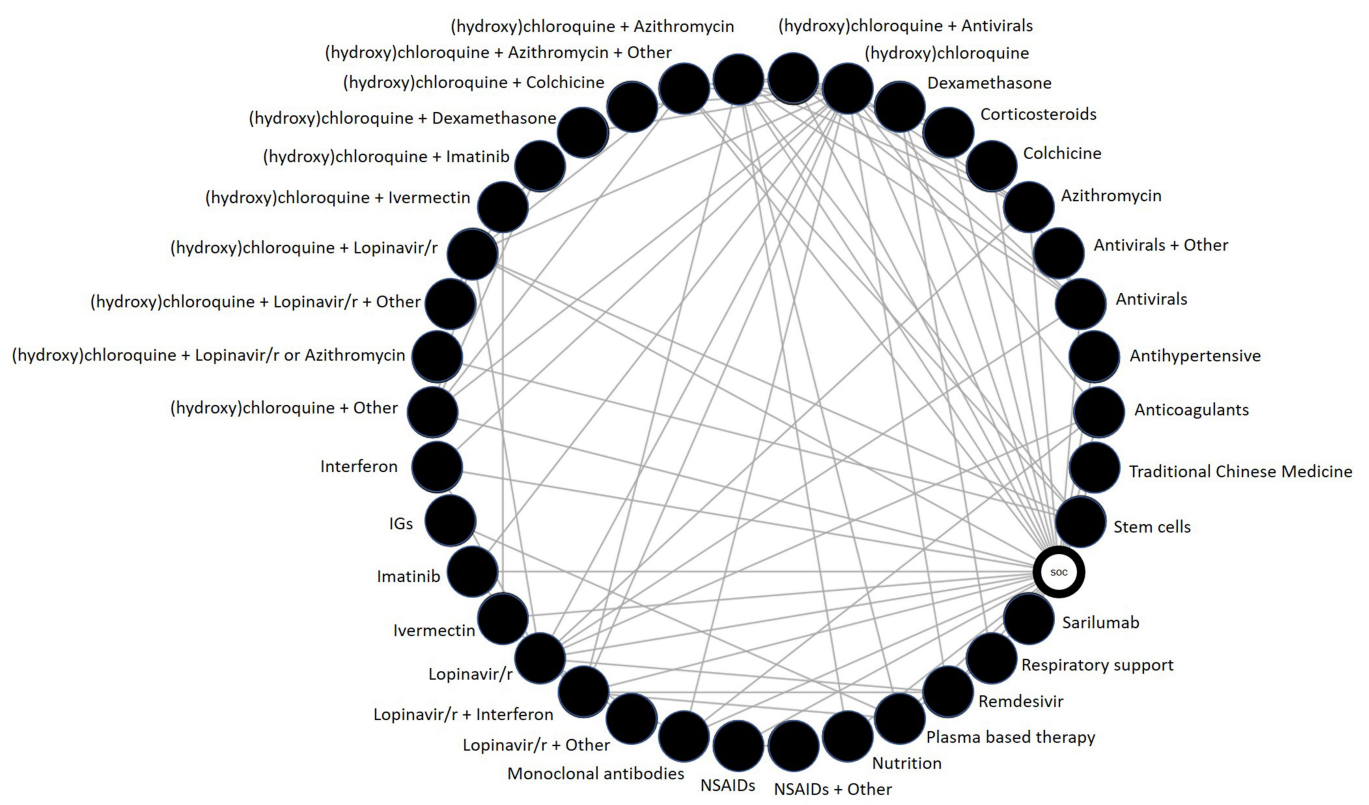

Figure 6 Treatment network for (A) outpatient and (B) hospitalized or intensive care unit randomized clinical trials for COVID-19. Notes: Each node (circle) represents an intervention and each line represents a direct comparison between different interventions. Abbreviations: NSAIDs, nonsteroidal anti-inflammatory drugs; Lopinavir/r, lopinavir/ritonavir; IGs, immunoglobulin.

local standard of care remained the most common control $\operatorname{arm}(\mathrm{n}=206 / 266,63.66 \%)$. The median number of arms was two (IQR: 2, 2) across all trials and (hydroxy)chloroquine trials in the hospital or ICU setting (Figure 3).

Mortality was the most common outcome, listed in 719 (52.25\%) trials. Additional outcomes of common interest included invasive mechanical ventilation $(n=562 / 1376$,
40.84\%), adverse events ( $\mathrm{n}=408 / 1376,29.65 \%)$, and viral load or clearance $(n=289 / 1376,21.0 \%)$. The United States was the most common country listed in registry entries $(n=298 / 1376,21.66 \%)$, followed by Iran $(n=$ 212/1376, 15.41\%) and China $(\mathrm{n}=171 / 1376,12.43 \%)$. The planned median sample size across the hospitalized or ICU setting was 90 participants (IQR: 48, 200). 


\section{Discussion}

The planned evidence base for interventions to prevent and treat COVID-19 is rapidly expanding. To our knowledge, this is the first comprehensive landscape analysis of RCTs arranged by disease stages from prophylaxis to hospital or ICU admission. The vast majority of registered COVID-19 RCTs were based in the hospital or ICU setting, with a marked shortage of evidence anticipated to emerge from the PrEP, PEP, and outpatient settings. Consistent with sources reporting on COVID-19 publications, our results indicate the United States and China are among the countries with the highest number of registered RCTs at present. ${ }^{16,17}$ The preponderance of (hydroxy)chloroquine and two-arm trials across all disease stages suggests inefficiencies arising from duplicated treatments across trials and multiple control groups rather than a common control group used to evaluate different treatment arms. Within the context of a global pandemic, this speaks to opportunities for greater collaboration in clinical trial research to advance rapid scientific discovery; pooling resources into fewer clinical trials may provide a more efficient approach to COVID-19 RCTs. ${ }^{7,8,18-20}$

There is clinical value in classifying the progression of COVID-19 into stages. ${ }^{13,21,22}$ These delineations importantly highlight that results of clinical trials conducted in hospitalized or ICU settings may not necessarily generalize to those of PrEP, PEP, and outpatients, owing to significant differences in disease severity, level of healthcare attention necessary, and the types of interventions available to patients and clinicians. Certainly, there is an urgent need for trials of severely ill patients who require intensive care. $^{23}$ However, the public health benefit of reducing transmission and preventing hospitalization is also an essential area for continued research efforts. ${ }^{24}$

The results of our review indicate there are comparatively few studies in the prophylaxis and outpatient settings. Indeed, the vast majority of infected persons, including older adults and other high-risk populations, are managed in the outpatient setting. ${ }^{25}$ Yet, to date, no trial has definitively identified an effective PrEP, PEP, or outpatient treatment. ${ }^{26-29}$ Moreover, the long-term consequences of infection, including cases where hospitalization is not required, are not yet understood. The health, social, and economic impact of COVID-19 has become increasingly apparent, across public and population health, disruptions to supply chains and global financial markets, and at the individual level. ${ }^{30-34}$ Thus, evidence supporting prevention and early treatment is an area deserving of critical focus, with opportunities for rapid real-world application and an impact on the health and economic burden associated with COVID-19 on a global scale. ${ }^{30-34}$ There is a need for the scientific community to mobilize with trial efforts focusing on areas of high unmet need to generate meaningful evidence to advance the global health response to COVID-19..$^{6-8,18,19}$

This review was based on a robust and comprehensive approach to trial identification, which included drawing from several international clinical trial registries. The information from these trials was captured through piloted, standardized data extraction. However, there are limitations to our study. First, while this evidence is based on publicly available information contained in global clinical trial registries, research registration does not necessarily correlate perfectly to research activity. Trials may prospectively register ahead of trial initiation which may never occur. Second, there may be a lag between updates of trial status or registration. Importantly, not all countries have the same requirements for registration processes. Thus, it is possible that registrations and subsequent updates may be delayed or neglected. However, as our summary is intended to highlight general trends, rather than speak to the outcomes of individual studies, we do not anticipate a significant impact on our conclusions. Of note, important areas outside of the clinical trial context that are deserving of attention, including the role of social distancing measures and individual patient experiences, do not fall within the purview of our study on registered RCTs for COVID-19. Finally, our summaries inherently equate the quantity of emerging evidence with its quality as we suggest that more research into a clinical context will be more likely to generate useful findings. However, as there is no effective intervention yet identified for persons in the PrEP, PEP, or outpatient setting, we maintain that our critique of this imbalance in research efforts remains valid.

\section{Conclusion}

We identified an important gap in the emerging evidence for COVID-19, where comparatively few RCTs are focused on the PrEP, PEP, and outpatient settings. Across all disease stages, trials most often were two-armed, US-based, and included (hydroxy)chloroquine. Our results indicate the majority of registered RCTs are in the hospital or intensive care setting; thus, significant attention has been given to identifying interventions to support clinicians treating 
patients in advanced disease stages. However, effective intervention in earlier disease stages, including in the prevention of infection, may realize important downstream benefits for both patients and the broader healthcare system by mitigating the risk of hospitalization and death.

\section{Ethics Statement}

Not applicable. This is a review of registered clinical trials.

\section{Author Contributions}

All authors made a significant contribution to the work reported, whether that is in the conception, study design, execution, acquisition of data, analysis and interpretation, or in all these areas; took part in drafting, revising or critically reviewing the article; gave final approval of the version to be published; have agreed on the journal to which the article has been submitted; and agree to be accountable for all aspects of the work.

\section{Funding}

The Bill \& Melinda Gates Foundation sponsored this study.

\section{Disclosure}

The authors report no conflicts of interest for this work.

\section{References}

1. Sohrabi C, Alsafi Z, O'Neill N, et al. World Health Organization declares global emergency: a review of the 2019 novel coronavirus (COVID-19). Int J Surg. 2020;76:71-76. doi:10.1016/j.ijsu.2020.02.034

2. Fauci AS, Lane HC, Redfield RR. Covid-19-navigating the Uncharted. Mass Medical Soc; 2020.

3. Sanders JM, Monogue ML, Jodlowski TZ, Cutrell JB. Pharmacologic treatments for coronavirus disease 2019 (COVID-19): a review. JAMA. 2020;323(18):1824-1836.

4. Thorlund K, Dron L, Park J, Hsu G, Forrest JI, Mills EJ. A real-time dashboard of clinical trials for COVID-19. Lancet Digit Health. 2020;2(6):e286-e287. doi:10.1016/S2589-7500(20)30086-8

5. McCoy D, Chand S, Sridhar D. Global health funding: how much, where it comes from and where it goes. Health Policy Plan. 2009;24 (6):407-417. doi:10.1093/heapol/czp026

6. Bauchner H, Fontanarosa PB, Golub RM. Editorial evaluation and peer review during a pandemic: how journals maintain standards. JAMA. 2020;324(5):453. doi:10.1001/jama.2020.11764

7. Bauchner H, Fontanarosa PB. Randomized clinical trials and COVID-19: managing expectations. JAMA. 2020;04:04.

8. Glasziou PP, Sanders S, Hoffmann T. Waste in covid-19 research. BMJ. 2020;369:m1847.

9. Collins FS, Morgan M, Patrinos A. The human genome project: lessons from large-scale biology. Science. 2003;300(5617):286-290. doi:10.1126/science.1084564

10. Adams MD, Kelley JM, Gocayne JD, et al. Complementary DNA sequencing: expressed sequence tags and human genome project. Science. 1991;252(5013):1651-1656. doi:10.1126/science.2047873
11. Collins FS, McKusick VA. Implications of the human genome project for medical science. JAMA. 2001;285(5):540-544. doi:10.1001/ jama.285.5.540

12. Ahn D-G, Shin H-J, Kim M-H, et al. Current status of epidemiology, diagnosis, therapeutics, and vaccines for novel coronavirus disease 2019 (COVID-19). J Microbiol Biotechnol. 2020;30(3):313-324. doi:10.4014/jmb.2003.03011

13. Gandhi RT, Lynch JB, Del Rio C. Mild or moderate COVID-19. $N$ Engl J Med. 2020;383(18):1757-1766. doi:10.1056/NEJMcp 2009249

14. R: A Language and Environment for Statistical Computing [computer program]. Vienna, Austria: R Foundation for Statistical Computing; 2013.

15. Salanti G, Kavvoura FK, Ioannidis JP. Exploring the geometry of treatment networks. Ann Intern Med. 2008;148(7):544-553. doi:10.7326/0003-4819-148-7-200804010-00011

16. Arab-Zozani M, Hassanipour S. Features and limitations of LitCovid hub for quick access to literature about COVID-19. Balkan Med J. 2020. doi:10.4274/balkanmedj.galenos.2020.2020.4.67

17. Siemieniuk RA, Bartoszko JJ, Ge L, et al. Drug treatments for covid-19: living systematic review and network meta-analysis. BMJ. 2020;370:m2980. doi:10.1136/bmj.m2980

18. Gates B. Responding to Covid-19-a once-in-a-century pandemic? $N$ Engl $J$ Med. 2020;382(18):1677-1679. doi:10.1056/NEJMp20 03762

19. Beaglehole R, Bonita R. Global public health: a scorecard. Lancet. 2008;372(9654):1988-1996. doi:10.1016/S0140-6736(08)61558-5

20. The RECOVERY Collaborative Group. Dexamethasone in hospitalized patients with Covid-19 - preliminary report. $N$ Engl $J$ Med. 2020:1-11. doi:10.1056/NEJMoa2021436

21. Cao X. COVID-19: immunopathology and its implications for therapy. Nat Rev Immunol. 2020;20(5):269-270. doi:10.1038/s415 77-020-0308-3

22. Liu Y, Yan L-M, Wan L, et al. Viral dynamics in mild and severe cases of COVID-19. Lancet Infect Dis. 2020.

23. Grein J, Ohmagari N, Shin D, et al. Compassionate use of remdesivir for patients with severe Covid-19. $N$ Engl J Med. 2020;382 (24):2327-2336. doi:10.1056/NEJMoa2007016

24. Pan A, Liu L, Wang C, et al. Association of public health interventions with the epidemiology of the COVID-19 outbreak in Wuhan, China. JAMA. 2020;323(19):1915-1923. doi:10.1001/jama.2020.6130

25. Garg S, Kim L, Whitaker M. Hospitalization rates and characteristics of patients hospitalized with laboratory-confirmed coronavirus disease 2019 - COVID-NET, 14 States, March 1 -30, 2020. MMWR Morb Mortal Wkly Rep. 2020;69:458-464. doi:10.15585/mmwr.mm6915e3

26. Boulware DR, Pullen MF, Bangdiwala AS, et al. A randomized trial of hydroxychloroquine as postexposure prophylaxis for Covid-19. $N$ Engl J Med. 2020;383(6):517-525. doi:10.1056/NEJMoa2016638

27. Skipper CP. Hydroxychloroquine in nonhospitalized adults with early COVID-19. Ann Intern Med. 2020;173:623-631. doi:10.7326/M204207

28. Mitjà O, Corbacho-Monné M, Ubals M, et al. Hydroxychloroquine for early treatment of adults with mild Covid-19: a randomized-controlled trial. Clin Infect Dis. 2020.

29. Cavalcanti AB, Zampieri FG, Rosa RG, et al. Hydroxychloroquine with or without azithromycin in mild-to-moderate Covid-19. $N$ Engl J Med. 2020;383(21):2041-2052. doi:10.1056/NEJMoa2019014

30. Nicola M, Alsafi Z, Sohrabi C, et al. The socio-economic implications of the coronavirus and COVID-19 pandemic: a review. Int J Surg. 2020;78:185-193. doi:10.1016/j.ijsu.2020.04.018

31. Baker SR, Bloom N, Davis SJ, Terry SJ. Covid-Induced Economic Uncertainty. National Bureau of Economic Research; 2020:0898-2937.

32. Ma X, Vervoort D, Reddy CL, Park KB, Makasa E. Emergency and essential surgical healthcare services during COVID-19 in low-and middle-income countries: a perspective. Int J Surg. 2020;79:43-46. doi:10.1016/j.ijsu.2020.05.037 
33. Miller IF, Becker AD, Grenfell BT, Metcalf CJE. Disease and healthcare burden of COVID-19 in the United States. Nat Med. $2020 ; 1-6$.
34. McKee M, Stuckler D. If the world fails to protect the economy, COVID-19 will damage health not just now but also in the future. Nat Med. 2020;26(5):640-642. doi:10.1038/s41591-020-0863-y

\section{Publish your work in this journal}

Infection and Drug Resistance is an international, peer-reviewed openaccess journal that focuses on the optimal treatment of infection (bacterial, fungal and viral) and the development and institution of preventive strategies to minimize the development and spread of resistance. The journal is specifically concerned with the epidemiology of

Submit your manuscript here: https://www.dovepress.com/infection-and-drug-resistance-journa antibiotic resistance and the mechanisms of resistance development and diffusion in both hospitals and the community. The manuscript management system is completely online and includes a very quick and fair peerreview system, which is all easy to use. Visit http://www.dovepress.com/ testimonials.php to read real quotes from published authors. 